\title{
Bridging clinical perspectives with population health research
}

\author{
Jeff A. Dennis PhD
}

\begin{abstract}
Interdisciplinary research spanning clinical and population health perspectives has the potential to explore research areas that might not be examined within single respective disciplines. Barriers such as agreement on diagnostic criteria for identifying analytical subgroups of interest may inhibit both the initiation and the progress of this type of research. This column explores this type of collaboration via the methods and decision criteria used in a study on hypothyroidism and sleep apnea in a large sample of U.S. adults. Despite the barriers, these types of collaborations stand to inform clinical and population research in the future, and although short term impact of interdisciplinary work may often be less than single discipline research, some research suggests that long-term impact may be more substantial.
\end{abstract}

Keywords: interdisciplinary research, population health, clinical research

Population health research using retrospective or archival data often lacks individual specificity in regard to helping health care practitioners in their daily decisions about individual patients. Similarly, clinical research may be difficult to translate to the population level due to methodological and economic challenges in collecting a large sample with adequate diagnostic criteria to identify subgroups by health condition. Despite these challenges, clinical and population health researchers collaborate frequently, although the methods and decisions that go into developing these collaborations are not always well understood.

The study of population health typically uses large samples or populations to understand health disparities or dynamics on a macro-level. This work tends to be at a lower cost than clinical research, although it is important to note that the National Center for Health Statistics operates on a roughly $\$ 160$ million budget every year to collect a variety of nationally representative datasets, provided to researchers mostly

Corresponding author: Jeff A Dennis

Contact Information: Jeff.dennis@ttuhsc.edu

DOI: 10.12746/swrccc.v7i31.599 free. ${ }^{1,2}$ Although using existing data has advantages in startup costs and time to analysis, a number of decisions throughout the process can have major implications on one's eventual results. In short, this type of research requires categorization of individuals based on common characteristics, including gender, race/ethnicity, and age. Although these groupings are reasonably standard, they contain their own subjectivities. For example, two individuals, aged 64 and 65 , may be grouped into "45-64" and "65+" age categories, respectively, obscuring age-specific similarities they share.

This article focuses more explicitly on the decisions needed in population research to define diagnostic groups for comparison. As an example, I examine a recent publication exploring the association between hypothyroidism and sleep apnea. ${ }^{3}$ The study is derived from the National Health and Nutrition Examination Survey (NHANES), a nationally representative sample of about 10,000 noninstitutionalized individuals collected every two years. Respondents answer a lengthy questionnaire, have a physical examination, and provide blood samples for a variety of lab tests. As such, NHANES provides a particularly large sample with the capability to explore 
group differences in clinical markers. Specifically, this study used NHANES 2007-08 because it was the only biennial data collection that included specific questions on sleep apnea and also tested for thyroid stimulating hormone (TSH).

First, sleep apnea was identified by self-reported doctor diagnosis (SRDD), "Has a doctor ever told you that you have a sleep disorder? If yes, what was it?" From a population health perspective, this question is useful because it provides an adequate sample of sleep apnea cases for statistical comparison $(N \approx 250)$. From a clinical perspective, this question is perhaps more daunting. We are not provided information on how the diagnosis was made, who specifically made the diagnosis, nor do we have any of the data points used to make the diagnosis. The de-identified nature of NHANES does not allow us to identify respondents for reasons of confidentiality, meaning there is no opportunity for follow-up or clarification. As such, we use the SRDD sleep apnea variable to define this group, knowing that it misses some cases of undiagnosed sleep apnea and could potentially include some individuals who misidentify sleep apnea as a prior diagnosis. Nonetheless, to compare at the population level, this group has value for empirical comparison.

Hypothyroidism is not a condition asked about specifically in the health questionnaire, although respondents are asked a more general "Have you ever been diagnosed with a thyroid condition?" This question has minimal value for identifying hypothyroidism, but fortunately, NHANES conducted TSH tests in most participants in 2007-08. As such, individuals with elevated TSH are identified by exceeding the lab-defined normal range of TSH $>5.6 \mathrm{mIU} / \mathrm{m}^{4}$ Secondly, NHANES provides a data file of all prescription medications the participant is currently taking. We identified individuals taking levothyroxine or desiccated thyroid as being persons with likely hypothyroid diagnoses. Together, elevated TSH and medication status are helpful toward establishing a hypothyroid group in NHANES. Realistically, these two data points would not be sufficient for a clinician to make a hypothyroidism diagnosis in an individual patient, but for a large dataset collected via a stratified random sample, these parameters are useful for establishing an estimate of the population with hypothyroidism.
We defined hypothyroid individuals as those taking hypothyroid medications, those with $\mathrm{TSH}>5.6$ $\mathrm{mIU} / \mathrm{m}$, or both. The value of NHANES, different from a smaller clinical sample of individuals with confirmed hypothyroidism, is that these parameters allow for the estimation of a few different subgroups in the broader U.S. adult population. "Undiagnosed hypothyroidism" was defined as individuals with elevated TSH, not on thyroid medication. "Controlled hypothyroidism" was defined as individuals on medication with TSH levels in the normal range. Finally, "uncontrolled hypothyroidism" was defined as individuals with elevated TSH who were currently taking thyroid medication. The study found that as much as $2.4 \%$ of the U.S. adult population may have undiagnosed hypothyroidism. ${ }^{3}$ Among NHANES's greatest strengths is its capacity for identifying the prevalence of undiagnosed conditions in the population, and although these parameters would not be enough for a clinical hypothyroid diagnosis, the findings provide useful clinical and public health insight relating to the estimated prevalence of an undiagnosed condition.

Ultimately, our results found a positive association between hypothyroidism and sleep apnea, although primarily among those taking hypothyroid medication. These results should not be interpreted as thyroid medication's causing sleep apnea, but rather may highlight unmeasured aspects of health care access. Although we control for self-reported health care access, those taking thyroid medication likely visit a general practitioner and possibly an endocrinologist multiple times per year. Increased frequency of health care interactions may also increase the likelihood of being screened for comorbid conditions or reporting other symptoms to one's physicians. We are not able to control for the frequency of regular health care visits, so we are left to speculate on the nature of the association. For example, the endocrinologist might routinely screen all hypothyroid patients for sleep apnea, whereas the family practitioner might not. Access to an endocrinologist might be associated with access to diagnostic sleep studies.

The future of interdisciplinary research that combines clinical and population perspectives is promising, yet barriers remain. Interdisciplinary work tends to be cited less in the short term relative to single 
discipline research, but may accrue more citations in the long term. ${ }^{5}$ Research of this nature may have limited direct applications for clinical practice, as the relationship between hypothyroidism and sleep apnea is an association rather than a causal link. However, using population data to explore associations among different clinical diagnoses may serve to open new doors of inquiry for future clinical and population health research. Without collaboration between clinical and population health researchers, these two areas may operate in mutually exclusive circles, contributing to segmented and at times uninformed research outputs.

Article citation: Dennis JA. Bridging clinical perspectives with population health research. The Southwest Respiratory and Critical Care Chronicles 2019;7(31):56-58

From: Department of Public Health, Texas Tech University Health Sciences Center, Lubbock, Texas

Submitted: 9/23/2019

Accepted: 9/26/2019

Reviewer: Gilbert Berdine MD

Conflicts of interest: none

This work is licensed under a Creative Commons Attribution-ShareAlike 4.0 International License.

\section{REFERENCES}

1. National Center for Health Statistics. About the budget. https://www.cdc.gov/nchs/about/budget.htm. Accessed 18 August 2019.

2. Dennis JA. Using 'Big Data' to Improve Health Care Services and Research. The Southwest Respiratory and Critical Care Chronicles 2016;4(13):49-51.

3. Thavaraputta S, Dennis JA, Laoveeravat P, Nugent K, Rivas AM. Hypothyroidism and its association with sleep apnea among adults in the United States: NHANES 2007-2008. J Clin End Met 2019;104(11)4990-4997.

4. Laboratory Procedure Manual. Available: https://wwwn. cdc.gov/nchs/data/nhanes/2007-2008/labmethods/thyrod_e_ met_thyroid_stimulating_hormone.pdf. Accessed 15 August 2019.

5. Van Noorden R. Interdisciplinary research by the numbers. Nature 2015;525:306-7. 